\title{
ALCOHOL ALERT
}

\section{A Developmental PERSPECTIVE ON Underage AlCOHOL UsE}

Dramatic developmental changes unfold as individuals mature from birth to childhood, from childhood to adolescence, and from adolescence to early adulthood. These include physiological changes — such as physical growth, brain development, and puberty — as well as psychological and social changes - such as an evolving sense of self, forming more mature relationships with friends, and transitioning from middle school to high school.

Developmental changes factor into underage drinking. For example, as a high school student transitions to college, he or she may experience greater freedom and autonomy, creating more opportunities to use alcohol. Underage drinking also can influence development, potentially affecting the course of a person's life. For example, alcohol use can interfere with school performance and/or negatively affect peer relationships.

This Alcohol Alert examines the complex relationship between underage drinking and development: how developmental factors influence drinking, the social and physical consequences of alcohol use, and how various developmental stages can be specifically targeted to design more effective measures for preventing or treating underage drinking.

\section{Key Stages in Human DEVELOPMENT}

As children mature, they achieve key developmental milestones such as changing the way they relate to parents and peers, beginning school and moving through different grades and school settings, undergoing puberty, gaining greater independence, and taking on more responsibilities. These milestones may come earlier for some individuals than others, depending on how quickly they mature, but tend to correspond, in general, to certain ages and developmental stages:

- Prenatal: prior to birth

- Ages 0-10: childhood

- Ages 10-15: early to mid-adolescence

- Ages 16-20: late adolescence

- Ages 21-25: transition to early adulthood

Each stage in development carries risks for alcohol use and its consequences. Studies show that alcohol use typically begins in early adolescence (ages 12-14) (1) and that between ages 12 and 21 , rates of alcohol use and binge drinking ${ }^{1}$ increase sharply before leveling off in the twenties (see figure, page 3) (2).

\section{How Developmental Factors INFLUENCE DRINKING AND RISK FOR DRINKING}

Researchers are investigating the complex relationship between developmental factors and alcohol use to better understand how the risks for drinking and alcohol-related problems emerge across development.

Personality and Behavior. Aspects of personality/temperament and certain behaviors that are evident early in life-often before children enter elementary school — such as antisocial behavior, poor self-regulation, poor self-control, anxiety, a tendency toward depression, and shyness may predict initiation of alcohol use in early adolescence, as well as future heavy use and alcohol use disorders (AUDs) $(3,4)$. Individuals with the most persistent personality and behavior problems are those most likely to experience more chronic and severe forms of AUDs in adulthood (5).

Family Dynamics. Family dynamics also factor into a child's risk for underage drinking. When parents respond well to their child's needs,

\footnotetext{
${ }^{1}$ Binge drinking is defined as consuming five or more drinks in a row for men and four or more drinks in a row for women. Source: http://www.collegedrinkingprevention.gov.
} 
that child is better able to regulate his or her emotions and behavior (6). The most effective family environments are characterized by greater warmth, moderate discipline, and limited stress (7). Conversely, parents who are depressed, antisocial, or aggressive toward their children or who create a family atmosphere marked by conflict may hinder their child's ability to regulate and control his or her own behavior (8). Problems with behavioral control, in turn, increase the risk for involvement with alcohol and other drugs (AODs). Early exposure to AOD use by parents and siblings also increases the risk for underage alcohol use.

Peer Relationships and Culture. As they mature, adolescents place increased importance on peer relationships. Peers who drink may encourage experimentation with alcohol use (9). This experimentation can have potentially dangerous consequences. Yet, as researchers note, the increased risk-taking and experimentation that is characteristic of adolescence marks an important developmental progression, as individuals begin to form their own identities and to develop strong bonds with peers (10).

Gene-Environment Interaction. Research suggests that the interaction of inherited and environmental factors strongly influences drinking behavior and that their relative influence varies across adolescence (11). For example, the initiation of alcohol use is tied more to environmental than to genetic influences. In contrast, across mid- to late adolescence, the relative influence of genetic factors on underage drinking increases, although there are important individual differences (12). Some of the genetic factors that influence problem drinking are specific to alcohol, whereas others influence a range of behaviors that reflect a general lack of impulse control in late adolescence and early adulthood (13).

\section{Adolescent Brain Development and Gaps in Maturity.}

All regions of the brain do not mature at the same time or at the same pace. For example, a region deep within the brain that governs emotions and mediates fear and anxiety (i.e., the limbic system) matures in early adolescence. Its development is believed to be triggered by the hormones that set puberty in motion. In contrast, the frontal cortexthe region responsible for self-regulation, decisionmaking, and behavioral control — develops more gradually, as a result of age and experience. This creates a period of time during adolescence in which emotions are heightened, but the ability to regulate these emotions and regulate one's behavior still is developing. Some researchers believe this differential maturation of brain regions may contribute to the increased risk-taking behavior common during adolescence (14-16).

Differences in Sensitivity to Alcohol. Research with animals suggests that compared with adults, adolescents are less sensitive to the negative effects of alcohol intoxicationsuch as sedation, hangover, and loss of coordination-but are more sensitive to the way alcohol eases social situations (17). Because human adolescents may be less sensitive than adults to certain aversive effects of alcohol, they may be at higher risk for consuming more drinks per drinking occasion. This developmental phenomenon may help explain why adolescents are able to drink larger amounts of alcohol (as in binge drinking) without experiencing the same levels of physiological effects (such as sleepiness and poor coordination) as adults.

\section{How Drinking CAN INFLUENCE DEVELOPMENT}

The immediate, short-term, and long-term effects of alcohol vary with developmental period.

Prenatal. The developing embryo and fetus are particularly vulnerable to the adverse effects of alcohol. Prenatal exposure to alcohol can result in a wide range of physical abnormalities, growth retardation, and nervous system impairments (collectively referred to as Fetal Alcohol Spectrum Disorder; the most severe form of this disorder is called Fetal Alcohol Syndrome) (18).

Childhood and Adolescence. Underage drinking can interfere with school attendance, disrupt concentration, damage relationships with parents and peers, and potentially alter brain function and/or other aspects of development, all of which have consequences for future success in such areas as work, adult relationships, health, and overall well-being (19).

A person who begins drinking early in life also is more likely to become a heavy drinker during adolescence and to experience alcohol abuse or dependence on alcohol in adulthood $(20,21)$.

In addition, chronic heavy drinking during adolescence has been linked to cognitive deficits and alterations in brain activity and structure $(22,23)$. It is unclear, however, whether these deficits resulted from alcohol consumption itself or existed prior to the initiation of drinking and may in fact have contributed to that individual's alcohol use (24). It is also unknown if and to what extent these deficits will resolve with abstinence or decreased drinking. Human studies (25-27) have shown, however, that adolescents are more vulnerable than adults to alcohol-related effects on memory. Additionally, evidence from rodent studies of binge-type drinking suggests that adolescent animals are more vulnerable than adults to brain damage (28).

Late Adolescence. Across multiple studies, there seems to be a strong relationship between drinking in later adolescence and in early adulthood. Research also shows that people who drink heavily in late adolescence are more likely than others to be diagnosed with an AUD later in life (29). In addition, alcohol use in late adolescence is associated with a 


\section{Past-Month Alcohol Use (any, binge, heavy) by Age}

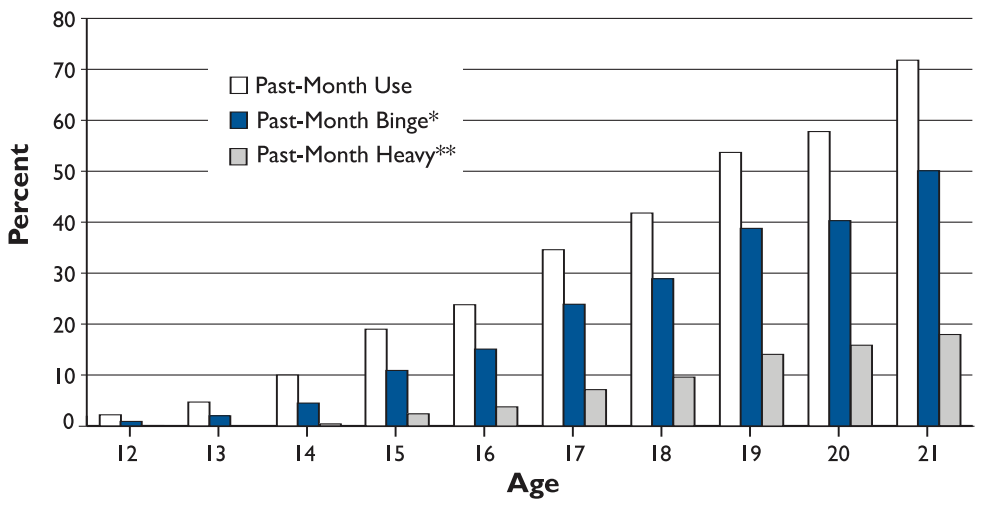

NOTE: *Binge is defined as five or more drinks on an occasion.

**Heavy Drinking is defined as five or more drinks on an occasion on five or more of the past 30 days.

SOURCE: SAMHSA, National Survey on Drug Use and Health, 2007. shown effectiveness in delaying the initiation of alcohol use and reducing drinking during the teenage years.

For adolescents aged 10-15, family-focused interventions have shown considerable promise (32). Interventions for this age-group can be either home based or administered in small groups, and analysis indicates that small-group interventions show relatively stronger evidence of effect than home-based interventions. Family-focused interventions have not shown this same degree of effectiveness for older adolescents who do not enter college, although studies focusing on college-bound adolescents have shown some success (33).

School-Based Interventions. Unlike familybased approaches, which tend to focus on strengthening parenting skills and parent-

number of other serious problems in adulthood, including drug dependence, antisocial behavior, and depression (29-31), although evidence of this latter association has been inconsistent (31).

\section{Prevention}

The harmful consequences of alcohol use underscore the importance of evidence-based preventive interventions and policy measures for preventing underage drinking. Preventive interventions may target every member of an eligible population or a particular subpopulation with a higher risk of problems, including AUDs. Findings from a recent review by Spoth and colleagues (32) of relevant intervention studies are summarized below.

Family-Focused Interventions. These interventions typically address a range of risk and protective factors associated with life within a family. For instance, do parents carefully monitor the child's activities, are they "close" (i.e., bonded) to the child, do they practice effective discipline, and are they involved in the child's home life and school? Many familyfocused interventions have been aimed at families with preschool-aged children and have focused on improving parent-child relationships, decreasing aggressive behavior, and enhancing the children's social and psychological readiness to make the transition to school. Most studies evaluating these interventions have concentrated on the effectiveness of these measures in curbing aggressive behavior. Only one preschool program evaluated alcohol use later in life and showed reduced drinking by participants when they reached their teen years (32).

Few family-focused interventions for elementary schoolaged children have been implemented and assessed, although interventions for this population often have family and school components (32). Several of these interventions have child relationships, school-based approaches focus on life skills, peer refusal skills, role-playing, strengthening positive peer relationships, and providing accurate information on how many children actually use alcohol. Such interventions have been shown to significantly reduce aggressive and disruptive behavior in younger children, as well as early initiation and progression of alcohol use in adolescents (32). However, many studies have important limitations, including not following children long enough to evaluate eventual alcohol use. Among the programs reviewed, there were no effective interventions with children in later elementary school years (i.e., grades 3 to 5) with respect to early alcohol use. Similarly, the researchers found only two promising schoolbased interventions targeted to high school students (32).

Multidomain Interventions. As the name implies, these interventions address several aspects of an adolescent's life (e.g., the individual, family, school, and community/ environment) and typically focus on younger adolescents. Research indicates that these interventions can be effective, but there is a need for further research to develop and assess such interventions for older adolescents (34). In addition, there are challenges to large-scale implementation of these programs, including the extensive resources required to sustain quality implementation (32).

\section{Policy-, Law-, and Environmentally Focused}

Interventions. These types of interventions typically focus on older adolescents. The review by Spoth and colleagues (32) did not find any effective policy interventions aimed at adolescents younger than age 16, nor did it identify policy interventions that could be shown to delay initiation of alcohol use or reduce alcohol use among younger adolescents. For older adolescents, several interventions have shown promise, including those focused on reducing the sale of alcohol to minors, increasing identification checks ("carding") by vendors, and reducing the community's tolerance of the 
sale of alcohol to minors and of underage drinking in general. However, studies assessing the effectiveness of these measures often did not investigate alcohol use outcomes specifically, or they involved so few communities that it is impossible to say if these measures would be effective for all communities.

One policy that has been extensively studied is the effectiveness of laws raising the minimum legal drinking age from 18 to 21. Most studies have found a positive effect on health and safety when the minimum drinking age is increased to 21. Studies also have shown that changes in the drinking age laws can reduce the rates of underage drinking (35), singlevehicle nighttime car crashes (the most common crash associated with alcohol use) (36), and vehicle fatalities (37). Other studies $(38,39)$, however, found no changes in the rates of crashes and fatalities after the introduction of such laws.

\section{TreatMenT}

Researchers have developed a variety of treatment approaches for adolescents with AOD use disorders. Medications rarely are used in this age-group, although some studies have examined the effects of certain drugs aimed at treating coexisting psychiatric conditions, such as depression or anxiety, in this population. For example, in one study of adolescents with bipolar disorder and an AOD use disorder, those who received lithium treatment were less likely to screen positive for AOD use and exhibited greater clinical improvement than did adolescents in a placebo group (40). Findings from a recent review by Deas (41) evaluating treatment in underage drinkers are summarized below.

Behavioral Therapy. This intervention attempts to identify the behaviors and situations in which AOD use occurs and then to disrupt those behaviors by equipping adolescents with skills to resist AOD use. It emphasizes stress management, assertiveness training, and self-control. One study of adolescents seeking treatment for AOD use disorders found that those receiving behavioral therapy reported less frequent drug use than adolescents in a comparison group (42). The treatment-seeking group also had improved school attendance and performance. Another study comparing behavioral therapy with supportive counseling in adolescents and adults found that those in the behavioral therapy group showed much greater reductions in AOD use than those in the supportive therapy group, both immediately after treatment and at a 9-month followup (43). The behavioral therapy group also showed greater improvements in other areas, including the number of days in school.

Cognitive-Behavioral Therapy (CBT). Built on basic behavioral therapy, CBT includes strategies to help the adolescent better understand the factors and situations that precede AOD use as well as the consequences of that use.

Kaminer and colleagues (44) compared CBT (i.e., presentations, modeling, role playing, and homework exercises) with traditional group therapy in a study of adolescents who abused
AODs in addition to having other psychiatric disorders. Adolescents in the CBT group achieved lower scores on the Teen Addiction Severity Index - a tool that assesses a variety of aspects (or domains) to determine the adolescent's need for treatment or counseling - than the comparison group.

\section{Family-Based Interventions and Multisystemic Therapy}

(MST). These approaches use family therapy principles to improve adolescent health-risk behaviors. MST provides intensive, home-based treatment addressing the child's environment, including his or her family, peer groups, school, and community. Research using an MST approach with juvenile offenders (which focused on changing behavior by identifying the strengths and weaknesses of each participant and forming an individualized treatment plan) found positive effects on several high-risk behaviors. Study participants were less likely to use AODs, to be arrested for AOD-related offenses, and to be convicted for aggressive criminal behaviors than a similar group that did not receive the intervention (45).

When family therapy was combined with CBT, adolescents who received the family therapy/CBT intervention, on average, were less likely to use alcohol during the 6-month follow-up period and reported lower marijuana use than did adolescents who received only a basic-information intervention (46).

Similarly, a study of 182 alcohol- and marijuana-abusing adolescents who received either multidimensional family therapy (designed to improve overall family functioning through multiple interventions in several key areas of life), adolescent group therapy, or a multifamily education intervention found that adolescents assigned to multidimensional family therapy showed the greatest improvement (compared with the other groups), with more than 40 percent of participants in this group reporting reduced AOD use, which persisted throughout the 1-year follow-up period (47).

Motivational Enhancement Therapy. Also known as motivational interviewing (MI), motivational enhancement therapy is designed to enhance a person's motivation to make changes regarding AOD use and to recognize and cope with those life situations that may trigger or sustain AOD use. MI is particularly appealing for treating AODabusing adolescents, who often do not seek treatment on their own and need to be motivated to do so and to change their behaviors. In one study, researchers found that students receiving a single, brief MI session during their freshman year in college significantly reduced their drinking and had fewer alcohol-related consequences at 6-month and 2-year followups than did students who did not receive the intervention (48). In another MI study, one session significantly reduced adolescents' nicotine, alcohol, and marijuana use at the 3-month followup, relative to those who received only basic AOD education (49). 


\section{Conclusion}

The developmental pathway from childhood to adulthood is marked by significant change. Studies examining the complex relationship between development and alcohol use help illustrate how a person's risk for drinking and alcoholrelated problems emerges across childhood, adolescence, and early adulthood. The consequences of drinking alcohol also vary with developmental period. These effects can be far-reaching, ranging from altered brain function, reduced academic and work performance, and increased risk of problems with alcohol and other psychiatric problems during adulthood, to increased risk for injury or death (e.g., from alcohol-related falls or traffic crashes).

Understanding the interaction between alcohol and development enables scientists to design better-targeted and more effective approaches for preventing underage alcohol use. Some of these approaches, which act at the level of the family, school, or multiple domains simultaneously, have been shown to be effective at least for certain age-groups in delaying the initiation of drinking and reducing the amount of alcohol consumed. Still, not all developmental stages have been equally explored, and population subgroups, such as certain rural, cultural, or ethnic populations, may not respond as well to existing interventions. More research is needed to determine what works and what doesn't in different settings.

Equally important, knowledge of the interrelationship between developmental stage and drinking behavior will help researchers and clinicians design more effective treatment programs for adolescents with AUDs that can effectively reduce alcohol consumption and its associated consequences.

\section{REFERENCES}

(1) Faden, V.B. Trends in initiation of alcohol use in the United States 1975 to 2003 Alcoholism: Clinical and Experimental Research 30:1011-1022, 2006. PMID: 16737460 (2) Substance Abuse and Mental Health Services Administration (SAMHSA) Results From the 2007 National Survey on Drug Use and Health: National Findings. NSDUH Series H-30, DHHS Publication SMA 06-4194. Rockville, MD: Substance Abuse and Mental Health Services Administration, 2008. (3) Caspi, A.; McClay, J.; Moffitt, T.E.; et al. Role of genotype in the cycle of violence in maltreated children Science 297:851-854, 2002. PMID: 12161658 (4) Mayzer, R.; Puttler, L.I.; Wong, M.M.; et al. Predicting early onset of first alcohol use from behavior problem indicators in early childhood. Alcoholism: Clinical and Experimental Research 26(Suppl.):124A, 2002. (5) Biglan, A.; Brennan, P.A.; Foster, S.L.; et al. Helping Adolescents at Risk: Prevention of Multiple Problem Behaviors. New York: Guilford, 2004. (6) Calkins, S.D. Origins and outcomes of individual differences in emotion regulation. Monographs of the Society for Research in Child Development 59:53-72, 1994. PMID: 7984167 (7) Shedler, J., and Block, J. Adolescent drug use and psychological health: A longitudinal inquiry. American Psychologist 45(5):612-630, 1990. PMID: 2350080 (8) Campbell, S.B.; Shaw, D.S.; and Gilliom, M. Early externalizing behavior problems: Toddlers and preschoolers at risk for later maladjustment. Developmental Psychopathology 12:467-488, 2000. PMID: 11014748 (9) Schulenberg, J.E., and Maggs, J.L. A developmental perspective on alcohol use and heavy drinking during adolescence and the transition to young adulthood. Journal of Studies on Alcohol Supplement (14):54-70, 2002. PMID: 12022730 (10) Maggs, J.L.; Almeida, D.M.; and Galambos, N.L. Risky business: The paradoxical meaning of problem behavior for young adolescents. Journal of Early Adolescence 15(3):344-362, 1995. (11) Rutter, M., and Silberg, J. Gene-environment interplay in relation to emotional and behavioral disturbance. Annual Review of Psychology 53:460-490, 2002. PMID: 11752493 (12) Rose, R.J.; Dick, D.M.; Viken, R.J.; and Kaprio, J. Geneenvironment interaction in patterns of adolescent drinking: Regional residency moderates longitudinal influences on alcohol use. Alcoholism: Clinical and Experimental Research 25(5):637-643, 2001. PMID: 11371711 (13) Krueger, R.F.; Hicks, B.M.; Patrick, C.J.; et al. Etiologic connections among substance dependence, antisocia behavior, and personality: Modeling the externalizing spectrum. Journal of Abnormal Psychology 111(3):411-424, 2002. PMID: 12150417 (14) Dahl, R.E. Adolescent Brain development: a period of vulnerabilities. Keynote address. Annals of the New York
Academy of Sciences 1021:1-22, 2004 PMID 15251869 (15) Romer, D., and Walker, E.F., eds. Adolescent Psychopathology and the Developing Brain. New York: Oxford University Press, 2007. (16) Steinberg, L.; Dahl, R.E.; Keating, D.; et al. Psychopathology in adolescence: Integrating affective neuroscience with the study of context. In: Cicchetti, D., Cohen, D., eds. Developmental Psychopathology, 2nd Ed. New York: Wiley, 2006. pp. 710-741. (17) Spear, L.P., and Varlinskaya, E.I. Adolescence: Alcohol sensitivity, tolerance, and intake. Recent Developments in Alcoholism 17:143-159, 2005. PMID: 15789864 (18) Jacobson, J.L., and Jacobson, S.W. Effects of prenatal alcohol exposure on child development. Alcohol Research \& Health 26:282-286, 2002. PMID: 12875038 (19) Masten, A.S.; Roisman, G.I.; Long, J.D.; et al. Developmental cascades: Linking academic achievement, externalizing and internalizing symptoms over 20 years. Developmental Psychology 41:733-746, 2005. PMID: 16173871 (20) Ellickson, P.L.; Tucker, J.S.; and Klein, D.J. Ten-year prospective study of public health problems associated with early drinking. Pediatrics 111:949-955, 2003. PMID: 12728070 (21) Hingson, R.; Heeren, T.; and Winter, M.R. Age at drinking onset and alcohol dependence: Age at onset, duration, and severity. Archives of Pediatrics and Adolescent Medicine 160:739-746, 2006. PMID: 16818840 (22) Tapert, S.F., and Schweinsburg, A.D. The human adolescent brain and alcohol use disorders. Recent Developments in Alcoholism 17:177-189, 2005. PMID: 15789866 (23) De Bellis, M.D.; Clark, D.B.; Beers, S.R.; et al. Hippocampal volume in adolescent-onset alcohol use disorders. American Journal of Psychiatry 157(5):737-744, 2000. PMID: 10784466 (24) Hill, S.Y. Trajectories of alcohol use and electrophysiological and morphological indices of brain development: Distinguishing causes from consequences. Annals of the New York Academy of Sciences 1021:245-259, 2004. PMID: 15251894 (25) Acheson, S.K.; Stein, R.M.; and Swartzwelder, H.S. Impairment of semantic and figural memory by acute ethanol: Age-dependent effects. Alcoholism: Clinical and Experimental Research 22(7):1437-1442, 1998. PMID: 9802525 (26) White, A.M., and Swartzwelder, H.S. Agerelated effects of alcohol on memory and memory-related brain function in adolescents and adults. Recent Developments in Alcoholism 17:161-176, 2005. PMID: 15789865 (27) Nagel, B.J.; Schweinsburg, A.D.; Phan, V.; and Tapert, S.F. Reduced hippocampal volume among adolescents with alcohol use disorders without psychiatric comorbidity. Psychiatry Research 139(3), 181-190, 2005. PMID: 16054344 (28) Crews, F.T.; Braun, C.J.; Hoplight, B.; et al. Binge ethanol consumption causes differential brain damage in young adolescent rats compared with adult rats. Alcoholism: Clinical and Experimental Research 24(11):1712-1723, 2000. PMID: 11104119 (29) Wells, J.E.; Horwood, L.J.; and Fergusson, D.M. Drinking patterns in mid-adolescence and psychosocial outcomes in late adolescence and early adulthood. Addiction 99(12):1529-1541, 2004. PMID: 15585044 (30) Brook, D.W.; Brook, J.S.; Zhang, C.; et al. Drug use and the risk of major depressive disorder, alcohol dependence, and substance use disorders. Archives of General Psychiatry 59(11):1039-1044, 2002. PMID: 12418937 (31) Hill, K.G.; White, H.R.; Chung, I.J.; et al. Early adult outcomes of adolescent binge drinking: Person- and variable-centered analyses of binge drinking trajectories. Alcoholism: Clinical and Experimental Research 24(6):892-901, 2000. PMID: 10888080 (32) Spoth, R.; Greenberg, M.; and Turrisi, R. Preventive interventions addressing underage drinking: State of the evidence and steps toward public health impact. Pediatrics 121(Suppl. 4):S311-S336, 2008. PMID: 18381496 (33) Larimer, M.E., and Cronce, J.M. Identification, prevention, and treatment revisited: Individual focused college drinking prevention strategies 1999-2006. Addictive Behaviors 32:2439-2468, 2007. PMID: 17604915 (34) Perry, C.L.; Williams, C.L.; Komro, K.A.; et al. Project Northland: Longterm outcomes of community action to reduce adolescent alcohol use. Health Education Research 17(1):117-132, 2002. PMID: 11888042 (35) O'Malley, P.M., and Wagenaar, A.C. Effects of minimum drinking age laws on alcohol use, related behaviors and traffic crash involvement among American youth: 1976-1987. Journal of Studies on Alcohol 52(5):478-491, 1991. PMID: 1943105 (36) Hingson, R.W.; Scotch, N.; Mangoine, T.; et al. Impact of legislation raising the legal drinking age in Massachusetts from 18 to 20 . American Journal of Public Health 73(2):163-170, 1983. PMID: 6849474 (37) Decker, M.D.; Graitcer, P.L.; and Schaffner, W. Reduction in motor vehicle fatalities associated with an increase in the minimum drinking age. JAMA: Journal of the American Medical Association 260(24):3604-3610, 1988. PMID: 3193591 (38) Ruhm, C.J. Alcohol policies and highway vehicle fatalities. Journal of Health Economics 15(4):435-454, 1996. PMID: 10164038 (39) Vingilis, E., and Smart, R.G. Effects of raising the legal drinking age in Ontario. British Journal of Addiction 76(4):415-424, 1981. PMID: 6947816 (40) Geller, B.; Cooper, T.; Sun, K.; et al. Double-blind and placebo-controlled study of lithium for adolescent bipolar disorders with secondary substance dependence. Journal of the American Academy of Child and Adolescent Psychiatry 37(2):171-178, 1998. PMID: 9473913 (41) Deas, D. Evidence-based treatments for alcohol use disorders in adolescents. Pediatrics 121(Suppl. 4):S348-S354, 2008. PMID: 18381498 (42) Azrin, N.; Donohue, B.; Besalel, V.; et al. Youth drug abuse treatment: A controlled outcome study. Journal of Child and Adolescent Substance Abuse 3(3):1-16, 1994 (43) Azrin, N.; Acierno, E.; Kogan, E.; et al. Follow-up results of supportive versus behavioral therapy for illicit drug use. Behaviour Research \& Therapy 34(1):41-46, 1996. PMID: 8561763 (44) Kaminer, Y.; Burleson, J.; Blitz, C.; et al. Psychotherapies for adolescent substance abusers: A pilot study. Journal of Nervous and Mental Disorders 186(11):684-690, 1998. PMID: 9824170 (45) Henggeler, S.W.; Clingempeel, W.G.; Brondino, M.J.; and Pickrel, S.G. Four-year follow-up of multisystemic therapy with substance-abusing and substance-dependent juvenile offenders. Journal of the American Academy of Child and Adolescent Psychiatry 41(7):868-874, 2002. PMID: 12108813 (46) Latimer, W.W.; Winters, K.C.; D'Zurilla, T.; and Nichols, M. Integrated family and cognitive-behavioral therapy for adolescent substance abusers: A stage I efficacy study. Drug and Alcohol Dependence 71(3):303-317, 2003 PMID: 12957348 (47) Liddle, H.A.; Dakof, G.A.; Parker, K.; et al. Multidimensional family therapy for adolescent drug abuse: Results of a randomized clinical trial. American Journal of Drug and Alcohol Abuse 27(4):651-688, 2001. PMID: 11727882 (48) Marlatt, G.A.; Baer, J.S.; Kivlahan, D.R.; et al. Screening and brief intervention for high-risk college student drinkers: Results from a 2-year follow-up assessment. Journal of Consulting and Clinical Psychology 66(4):604-615, 1998. PMID: 9735576 (49) McCambridge, J., and Strang, J. The efficacy of single-session motivational interviewing in reducing drug consumption and perceptions of drug-related risk and harm among young people: Results from a multisite cluster randomized trial. Addiction 99(1):39-52, 2004. PMID: 14678061 


\section{Resources}

Source material for this Alcohol Alert originally appeared in Pediatrics: Official Journal of the American Academy of Pediatrics. April 2008, Volume I2I, Supplement 4.

For more information on underage drinking, see also:

Alcohol Research \& Health 32(I) focuses on underage drinking, and on how the process of human development (i.e., the changes that occur as young people age) influences underage drinking behavior. Articles identify biological and environmental risk factors that can make young people more likely to drink; describe the consequences of underage drinking; and evaluate prevention and treatment strategies that can help keep young people alcohol-free.

For these and other resources, visit NIAAA's Web site, www.niaaa.nih.gov.

Full text of this publication is available on NIAAA's World Wide Web site at www.niaaa.nih.gov.

All material contained in the Alcohol Alert is in the public domain and may be used or reproduced without permission from NIAAA. Citation of the source is appreciated.

Copies of the Alcohol Alert are available free of charge from the National Institute on Alcohol Abuse and Alcoholism Publications Distribution Center, P.O. Box 10686, Rockville, MD 20849-0686.

\section{U.S. DEPARTMENT OF}

\section{HEALTH AND HUMAN SERVICES}

NIAAA Publications Distribution Center

Attn.: Alcohol Alert

P.O. Box 10686

Rockville, MD 20849-0686

Official Business

Penalty for Private Use $\$ 300$
PRSRT STD

POSTAGE AND FEES PAID $\mathrm{NIH} / \mathrm{NIAAA}$

PERMIT NO. G-824 